Research Article

\title{
Numerical Investigation of Flow Field and Energy Loss in a Centrifugal Pump as Turbine
}

\author{
Jingze Li $\mathbb{D}^{\mathrm{D}}$, Dongrong Meng $\mathbb{D}$, and Xun Qiao \\ School of Mechanical Engineering, Xijing University, Xi'an 710123, China \\ Correspondence should be addressed to Jingze Li; ljz0624@126.com
}

Received 8 September 2020; Revised 31 October 2020; Accepted 6 November 2020; Published 26 November 2020

Academic Editor: Yong Zhu

Copyright (c) 2020 Jingze Li et al. This is an open access article distributed under the Creative Commons Attribution License, which permits unrestricted use, distribution, and reproduction in any medium, provided the original work is properly cited.

\begin{abstract}
Centrifugal pumps as turbine (PAT) are widely used in petrochemical and water conservancy industries. The research on the internal flow field and energy loss of PAT is of great significance to improve the performance and efficiency of PAT. In this paper, experimental and numerical simulation methods are used to study the energy loss and flow field. The results show that the numerical simulation method can accurately simulate the internal flow field of PAT. And the entropy generation theory is applied to visualize the internal energy loss of PAT through the comparison of total pressure loss and entropy generation. The highest energy loss among PAT components is the guide vane. The loss in the guide vane is mainly caused by the flow separation caused by the wake of the guide vane and the asymmetric structure of the volute. The losses in the impeller are mainly due to flow separation and wake. Besides, the unsteady simulation results show that rotor-stator interaction has a great influence on the gap between the impeller and the guide vane. The research results provide a reference for the design of the PAT. This study is beneficial to studying the dynamic and static interference and PAT vibration to improve the stability of the PAT.
\end{abstract}

\section{Introduction}

Centrifugal pumps as turbines (PAT) are widely used in steel, petrochemical, and water conservancy industries to recover energy from high-pressure liquids in the production process due to their low cost and high efficiency. PAT is in a long-term operating state, so it is of great significance to study the performance and efficiency of PAT [1-4].

At present, the methods of experiment and numerical simulation are used by most scholars to study the performance of PAT [5-10]. Bozorgi et al. [11] used numerical simulation to numerically simulate and verify the reverse mode industrial axial pump. The results show that the numerical simulation method can accurately predict the PAT performance and confirm that the axial flow pump in the reverse mode can operate normally in a small hydropower station. Su et al. [12] used the CFD method to numerically simulate the internal flow field of PAT and revealed the periodic law of flow velocity distribution in PAT. Wang et al. [13] used ANSYS and NX software to design a special forward-curved blade impeller. The comparison between the two impellers shows that the new impeller can significantly improve the efficiency of the PAT. Liu and Tan [14] conducted numerical simulation and experimental research on PAT with tip clearance. The results show that the increase of the blade tip clearance will cause the drop of PAT head and efficiency and reveal the vortex characteristics of PAT. Liu et al. [15] used the SST $k$ $\omega$ turbulence model to numerically simulate PAT. A new method to determine the best efficiency point (BEP) of PAT was proposed, and it is found that the high-efficiency area of PAT is relatively wide, which exists in large flow conditions. Tang et al. $[16,17]$ used machine learning methods to conduct in-depth research on fault diagnosis of rotating machinery. Gao et al. [18] discussed the numerical characterization of the influence of flow on the pressure and velocity distribution of PAT. Analyzing the internal flow phenomenon of PAT under different operating conditions, the results show that the unsteady turbulent flow in PAT will cause the vortex to have a greater impact on the flow separation [19].

Recently, the research on the internal flow of hydraulic machinery from the perspective of energy has attracted more and more attention from scholars [20-24]. Entropy 
generation theory has been applied in pipelines and pumps, but its application in PAT is also meaningful. In this paper, the entropy generation theory is used to analyze the energy loss characteristics in PAT. The SST $k$ - $\omega$ turbulence model is used to simulate the unsteady result of PAT and verify it by experiment. Based on the numerical simulation results verified by experiments, the energy loss distribution and formation mechanism inside the PAT are analyzed, and rotor-stator interaction phenomena in the PAT are analyzed.

\section{Entropy Production Theory}

The internal flow structure of PAT is complicated, and it is difficult to obtain detailed internal energy loss distribution using conventional methods. The entropy generation theory can be used to analyze the internal energy loss in detail. Entropy is one of the parameters that characterize the state of matter in thermodynamics; the balance equation (1) is the entropy in a single-phase incompressible fluid [25]:

$$
\rho\left(\frac{\partial s}{\partial t}+u_{1} \frac{\partial s}{\partial x}+u_{2} \frac{\partial s}{\partial y}+u_{3} \frac{\partial s}{\partial z}\right)=\operatorname{div}\left(\frac{\vec{q}}{T}\right)+\frac{\Phi}{T}+\frac{\Phi_{\Theta}}{T^{2}},
$$

where $s$ represents the specific entropy, $\Phi$ is the viscous dissipation term; and $\Phi_{\Theta}$ is the dissipation term caused by the heat transfer. The entropy production analysis is based on the second law of thermodynamics, combined with heat transfer theory and fluid mechanics to calculate the actual process dissipation of energy loss. Among them, the two rightmost items are entropy production items.

The temperature is set to a constant temperature in this paper, the entropy production item on the right side is processed by the Reynolds time-averaged process, and the local entropy production term expression is obtained as the two following equations:

$$
\begin{aligned}
& \dot{S}_{D}^{\prime \prime \prime}=2 \mu\left[\left(\frac{\partial u_{1}}{\partial x_{1}}\right)^{2}+\left(\frac{\partial u_{2}}{\partial x_{2}}\right)^{2}+\left(\frac{\partial u_{3}}{\partial x_{3}}\right)^{2}\right] \\
& +\mu\left[\left(\frac{\partial u_{2}}{\partial x_{1}}+\frac{\partial u_{1}}{\partial x_{2}}\right)^{2}+\left(\frac{\partial u_{3}}{\partial x_{1}}+\frac{\partial u_{1}}{\partial x_{3}}\right)^{2}+\left(\frac{\partial u_{2}}{\partial x_{3}}+\frac{\partial u_{3}}{\partial x_{2}}\right)^{2}\right] \\
& \dot{S}_{D^{\prime}}^{\prime \prime \prime}=2 \mu_{\mathrm{eff}}\left[\left(\frac{\partial u_{1}}{\partial x_{1}}\right)^{2}+\left(\frac{\partial u_{2}}{\partial x_{2}}\right)^{2}+\left(\frac{\partial u_{3}}{\partial x_{3}}\right)^{2}\right]+\mu_{\mathrm{eff}}\left[\left(\frac{\partial u_{2}}{\partial x_{1}}+\frac{\partial u_{1}}{\partial x_{2}}\right)^{2}+\left(\frac{\partial u_{3}}{\partial x_{1}}+\frac{\partial u_{1}}{\partial x_{3}}\right)^{2}+\left(\frac{\partial u_{2}}{\partial x_{3}}+\frac{\partial u_{3}}{\partial x_{2}}\right)^{2}\right], \\
& S_{\text {pro }, \bar{D}}=\int_{V} \dot{S}_{D}^{\prime \prime \prime} \mathrm{d} V \\
& S_{\text {pro }, \dot{D}}=\int_{V} \dot{S}_{D^{\prime}}^{\prime \prime \prime} \mathrm{d} V,
\end{aligned}
$$

where $S_{\text {pro }, \bar{D}}$ is the total entropy produced by the time-averaged velocity in the main flow area (W) and $S_{\text {pro, } D}$ is the total entropy production caused by the pulsation velocity in the main flow area $(\mathrm{W})$.

So, the total entropy production of the PAT is shown in the following equation:

$$
S_{\text {pro }}=S_{\text {pro }, \bar{D}}+S_{\text {pro }, \dot{D}}+S_{\text {pro }, W}
$$

The energy loss in the wall region (the area between the wall and the first layer of the grid) also occupies an important part. Since the entropy production in the wall could not be predicted precisely by these equations, the local entropy production rate for the wall is calculated using

$$
S_{\text {pro,W }}=\int_{A} \vec{\tau} \cdot \vec{v} \mathrm{~d} A
$$

where $\vec{\tau}$ is the wall shear stress $(\mathrm{Pa})$ and $\vec{v}$ is the velocity vector of the center of the first layer of the wall $(\mathrm{m} / \mathrm{s})$.

The total entropy produced by the mainstream area can be obtained by integrating the following equations:

\section{Numerical Model and Methods}

3.1. PAT Model. To analyze the internal flow characteristics and energy loss mechanism of PAT, a numerical simulation of a low specific speed single-stage centrifugal pump was carried out. The main parameters of the centrifugal pump are shown in Table 1. The PAT is composed of five main parts: inlet volute, guide vane, impeller, and export. The computational domain grids are all structured grids generated by commercial software ANSYS ICEM 18.0. Figure 1 shows the computational domain of the PAT model. To ensure accurate calculation of the boundary area, the 
TABLE 1: The main parameters of centrifugal pump.

\begin{tabular}{lcc}
\hline Parameter & & Value \\
\hline Discharge & $Q_{d}$ & $40 \mathrm{~m}^{3} / \mathrm{h}$ \\
Head & $H$ & $60 \mathrm{~m}$ \\
Rotating speed & $n$ & $2900 \mathrm{rpm}$ \\
Outer diameter of the impeller & $D 1$ & $223 \mathrm{~mm}$ \\
Impeller blade width & $b 1$ & $8 \mathrm{~mm}$ \\
Number of impeller blades & $z 1$ & 6 \\
Diameter of guide vane inlet & $D 2$ & $228 \mathrm{~mm}$ \\
Diameter of guide vane outlet & $D 3$ & $283 \mathrm{~mm}$ \\
Guide vane blade width & $b 2$ & $10 \mathrm{~mm}$ \\
Number of guide vanes & $z 2$ & 5 \\
Diameter of volute base circle & $D 4$ & $284 \mathrm{~mm}$ \\
\hline
\end{tabular}

boundary layer grid is encrypted and the $y^{+}$value is controlled to be less than 200, as shown in Figure 2 .

ANSYS CFX 18.0 was used for the steady and unsteady numerical simulation of PAT. The static pressure was set as the inlet boundary condition, the outlet boundary condition was set as the mass flow rate, all walls were set as nonslip wall surfaces, the frozen rotor is adopted at the rotor-stator interfaces, and the convergence criterion, RMS (root mean square), is set as $10^{-5}$. The turbulence model adopted the SST $k$ - $\omega$ turbulence model which was widely used in turbomachinery [27]. For unsteady numerical simulation, the time step was set to the time it takes for the impeller to turn $1^{\circ}$, which was $5.747 \times 10^{-5} \mathrm{~s}$. And, the steady simulation result is adopted to the initial value of the unsteady simulation.

Four grids with $4.7 \times 10^{6}, 6.57 \times 10^{6}, 8.05 \times 10^{6}$, and $9.4 \times 10^{6}$ were selected to validate the independence of the grid. Each set of grids operates under $0.8 Q_{d}, 1.2 Q_{d}$, and $1.6 Q_{d}$ conditions. The dimensionless pressure drop coefficient is calculated by the following equation:

$$
\varphi=\frac{P_{\mathrm{out}, t p}-P_{\mathrm{in}, t p}}{0.5 \rho u_{2}^{2}},
$$

where $P_{\text {out } t p}$ and $P_{\text {in }, t p}$ are the outlet and inlet pressures (Pa) and $u_{2}$ is the circumferential velocity of impeller tail edge $(\mathrm{m} / \mathrm{s})$. Table 2 shows the calculation result for different calculation grid numbers. The pressure drop coefficient calculated by the first three groups of grids has a significant increase, and the pressure drop coefficient calculated by the third and fourth groups of grids has little change. It shows that when the number of grids is greater than $8.05 \times 10^{6}$, the increase in the number of grids has little effect on the calculation results. So, to fully consider the computing resources to ensure the accuracy of the calculation, the fourth set of grids is selected for the numerical simulation of PAT.

3.2. Experimental Verification. The flow rate is measured by the Yokogawa AE215 flowmeter and the measurement error is $\pm 0.5 \%$. The pressure is measured by the Yokogawa EJA510A pressure sensor, and the measurement error is $\pm 0.075 \%$. JN338 type torque sensor is used to measure the torque, and its measurement error is $\pm 0.2 \%$. The unsteady flow field in PAT is studied and analyzed by numerical simulation. Figure 3 shows the test rig of model PAT and the comparison between numerical simulation results and experimental results. It can be seen that the numerical simulation results have the same trend as the experimental results, with the maximum error not exceeding 5\%. Figure 4 shows the comparison between the numerical simulation and the experiment of the pressure pulsation at the $P 3$ and $P 4$ monitoring points at $1.0 Q_{d}$. As can be seen from the figure, due to the effect of rotor-stator interaction, the pressure pulsation coefficient shows periodic fluctuations, and the time-domain curve distribution trend of numerical simulation of pressure pulsation is similar to the experiment. The time-domain distribution of the pressure pulsation at the monitoring point $P 3$ agrees well with the experimental value. The time-domain distribution of monitoring point $P 3$ is quite different from the experimental value. And the pressure pulsation coefficients at the monitoring points $P 3$ and $P 4$ are higher than the test values. However, the amplitude of the pressure pulsation in the numerical simulation is higher than the experimental value at both the leaf frequency and the harmonic frequency.

In summary, the monitoring positions can be accurately arranged during the numerical simulation. Moreover, factors such as gap leakage and disc friction loss were not considered in the numerical simulation process, so the numerical simulation pressure pulsation result was higher than the experimental value. But the distribution trend of numerical simulation results agrees well with the experiment. Therefore, the meshing, boundary conditions, and selection of turbulence models of each overcurrent component in the numerical method are reliable.

3.3. Entropy Generation Theory Verification. The total energy loss in PAT can be accurately obtained by the method of pressure drop. The entropy production theory can be used to analyze the internal loss of the PAT and its mechanism. The total entropy production can be calculated in equation (9) and the mechanical energy loss of the PAT can be calculated by the following equation:

$$
P=\rho g H Q .
$$

The runner shaft power can be calculated in the following equation:

$$
W_{\mathrm{sft}}=\frac{2 \pi \times M \times n}{60} .
$$




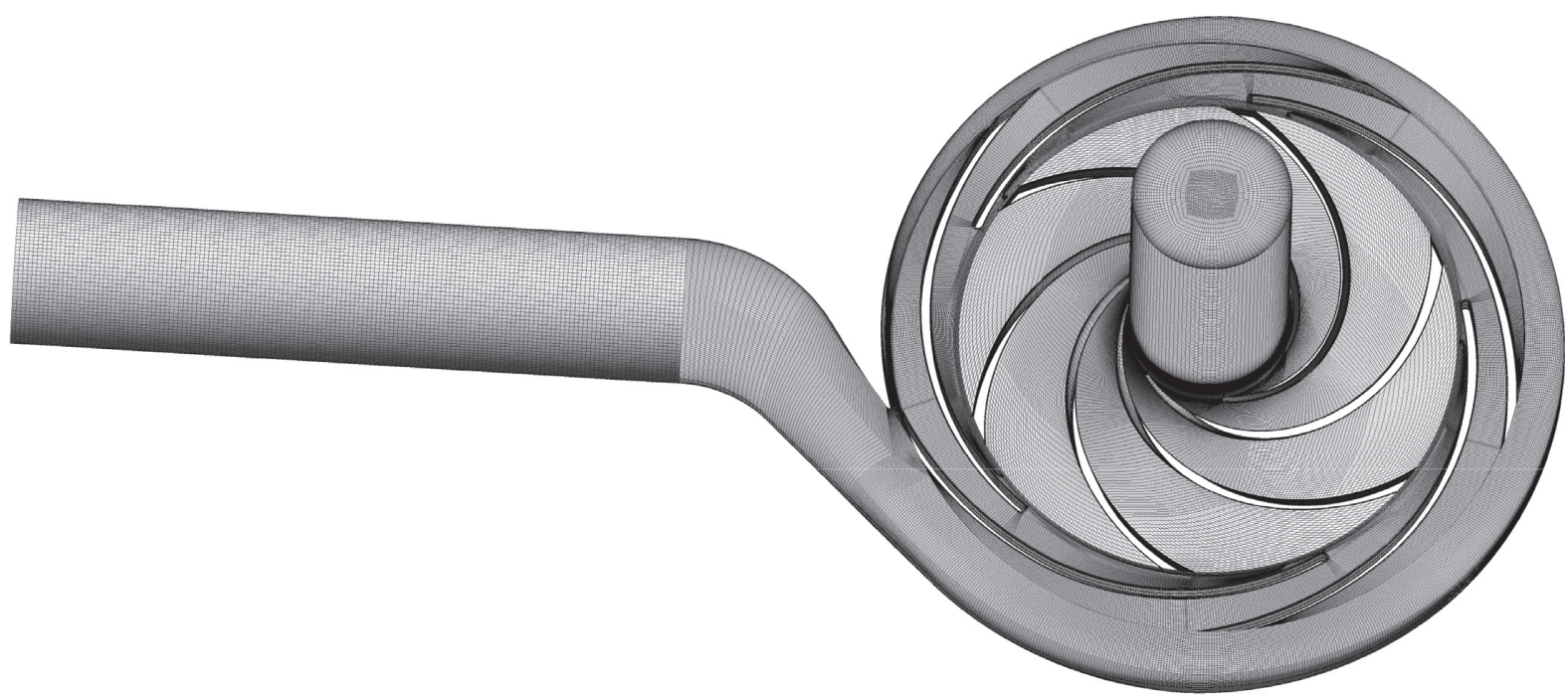

(a)

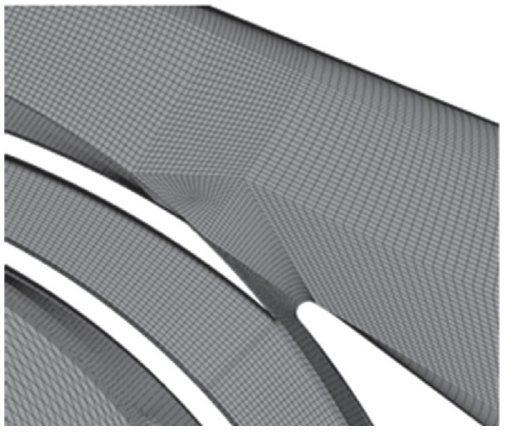

(b)

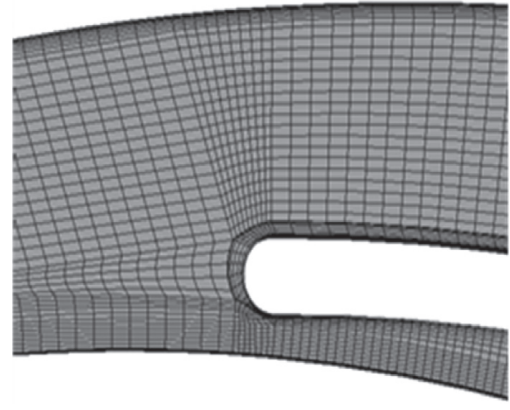

(c)

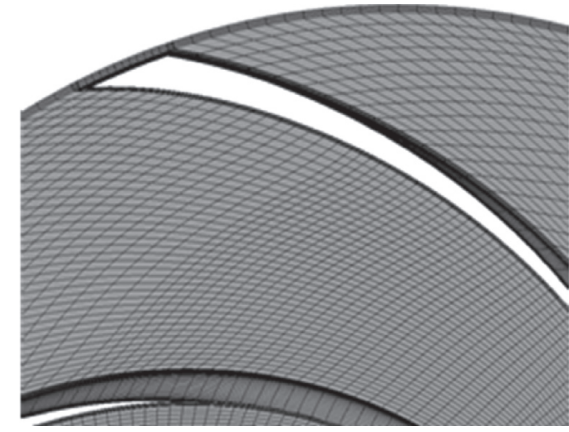

(d)

Figure 1: Computational domain of the PAT model.

So the accuracy of entropy production theory can be verified by these two equations, calculated as follows:

$$
P-W_{\text {sft }}=S_{\text {pro }} .
$$

The left side of the above equation is defined as $P_{\text {loss }}$, and the accuracy of entropy generation theory can be verified by comparing $P_{\text {loss }}$ and $S_{\text {pro }}$.

Figure 5 shows the energy loss of each component calculated by two different methods and the error of the results calculated by two methods. The energy loss calculated by the two methods tends to decrease first and then increase. The energy loss reaches the minimum value when $Q=40 \mathrm{~m}^{3} / \mathrm{h}$. This is because the design operating condition of the pump used as PAT is $Q=40 \mathrm{~m}^{3} / \mathrm{h}$. Under this working condition, the performance is the best and the internal flow is the most stable. At low flow rates, many vortices were generated in the PAT to block the flow passage, which caused higher losses. At the flow rates, the turbulence in the PAT was severe due to the high velocity, resulting in a high energy loss. The error between the entropy generation theory and the pressure drop method is less than $5 \%$ at $Q=48$. The error can reach up to $10 \%$ when the flow rate is small. This is because the flow is stable when the operating conditions are designed, and the small flow and large flow are complicated. The entropy generation theory still has errors in some complex flow situations. In general, the entropy generation theory can be used to predict the energy loss inside the PAT.

\section{Results and Discussion}

Figure 6 shows the identification of the blades and flow passages, among which the impeller blades are RB1, RB2, ..., RB6. The flow channel between the blades RB1 and RB2 is $\mathrm{RP} 1$. The naming rules in the guide vanes are similar to the impeller.

Figure 7 shows the total entropy production and wall loss under different flow conditions. The wall loss increases linearly with the increase in the flow rate, and the entropy production decreases first and then increases with the increase in the flow rate and reaches the minimum value at $Q_{d}$. This is because the fluid flow in the PAT is the best and the energy loss is the lowest at $Q_{d}$. Besides, the loss of entropy generation inside the PAT is higher than the loss near the wall.

Figure 8 shows the energy loss obtained by the pressure drop method among the three main components of the PAT over one impeller rotation period. The energy loss of each component shows obvious periodic fluctuations. There are 6 


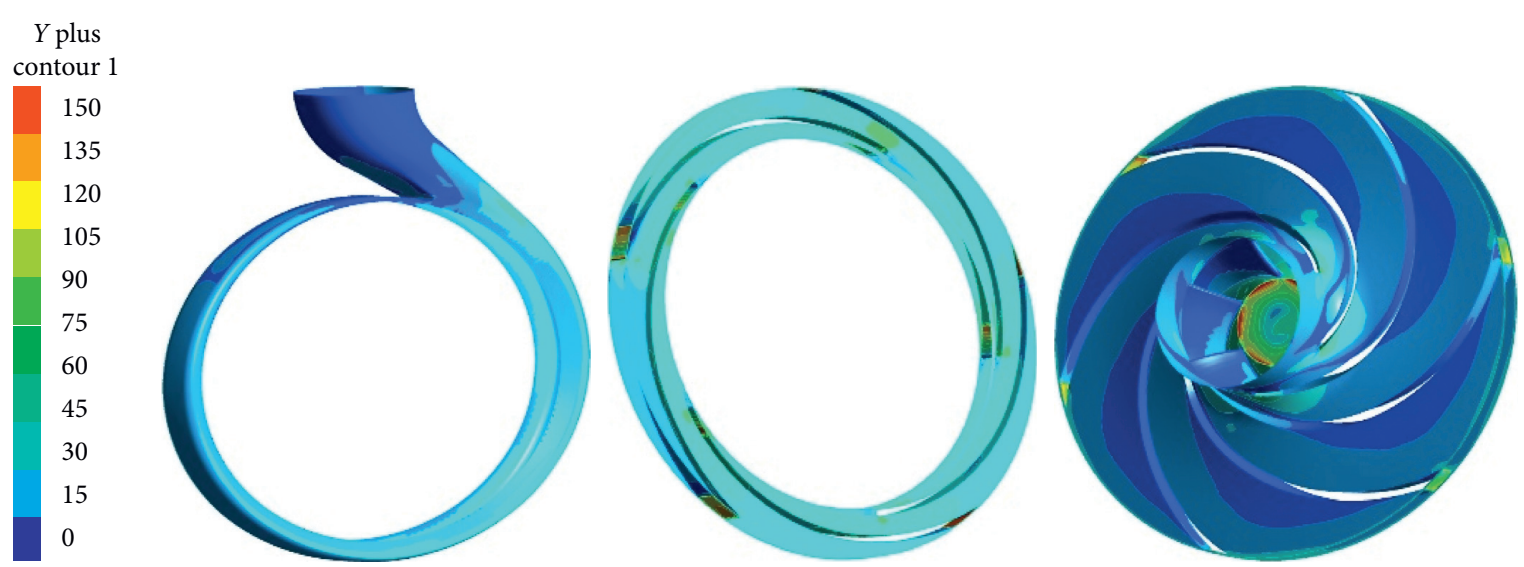

Figure 2: $y^{+}$at the wall in PAT.

TABle 2: Pressure drop coefficients of three groups of grids at different flow rates.

\begin{tabular}{llccr}
\hline$Q_{0}$ & & $G$ & & \\
& & & & \\
& $4.7 \times 10^{6}$ & $6.57 \times 10^{6}$ & $8.05 \times 10^{6}$ & 1.083976 \\
\hline 0.8 & 0.978976 & 1.078976 & 1.081976 & 1.494427 \\
1 & 1.441427 & 1.491427 & 2.133589 & 2.1356427 \\
\hline
\end{tabular}

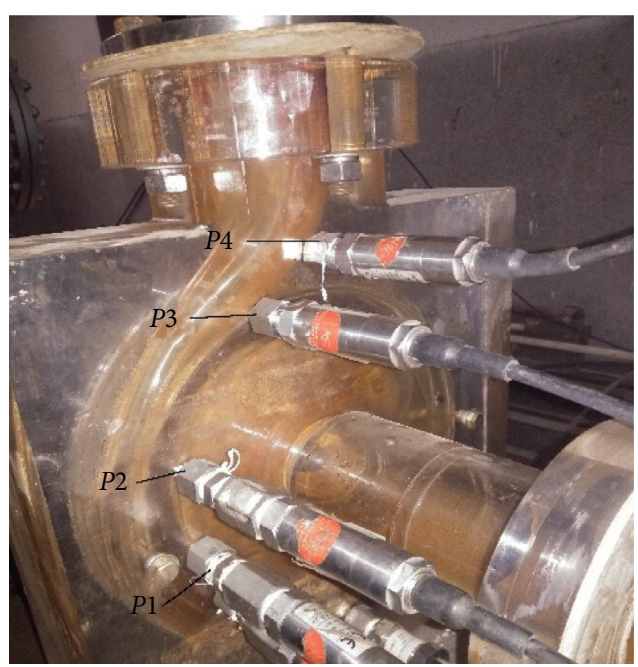

(a)

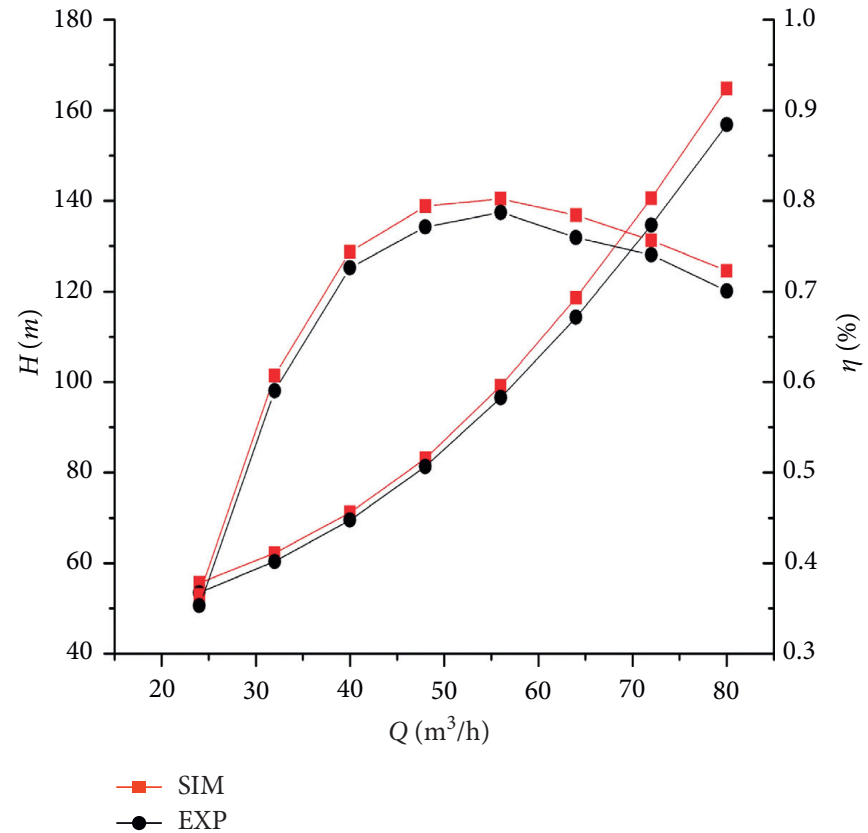

(b)

Figure 3: Test rig of model PAT and comparisons of experiments and simulations. (a) Test rig of model PAT. (b) Comparisons of experiments and simulations.

obvious wave crests and troughs on all three parts, which are the same as the number of impeller blades. And the loss of the three components reaches the crest and trough at the same time. Comparing the amplitude of the energy loss fluctuation of the three components, it is found that the amplitude of the loss in the impeller is the highest, and the amplitude of the loss in the volute is the lowest. Also, there are 5 smaller wave crests between the two wave crests of the impeller and the guide vane component, the number of which is the same as the number of the guide vane blades. The fluctuation between the two main wave crests of the volute component is not very obvious. This shows that the internal loss of PAT is affected by rotor-stator interaction, the impeller is most affected by rotor-stator interaction, and the volute is weakly affected by the interaction. 


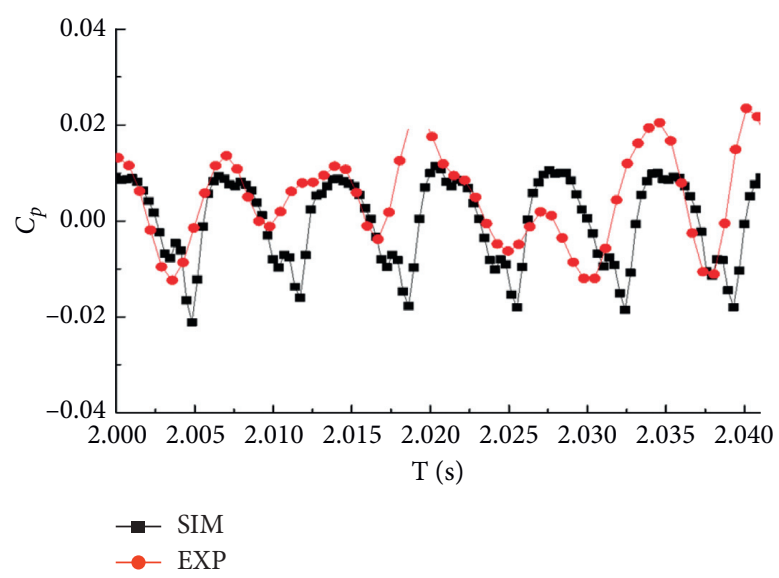

(a)

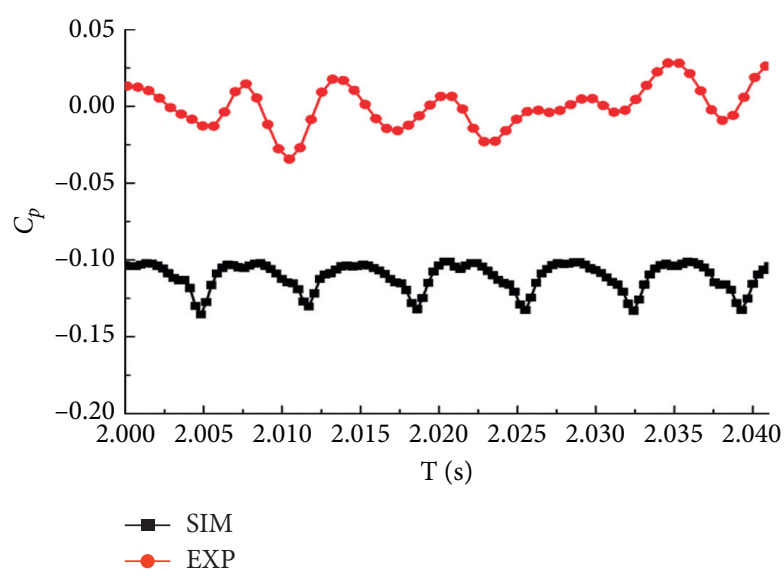

(c)

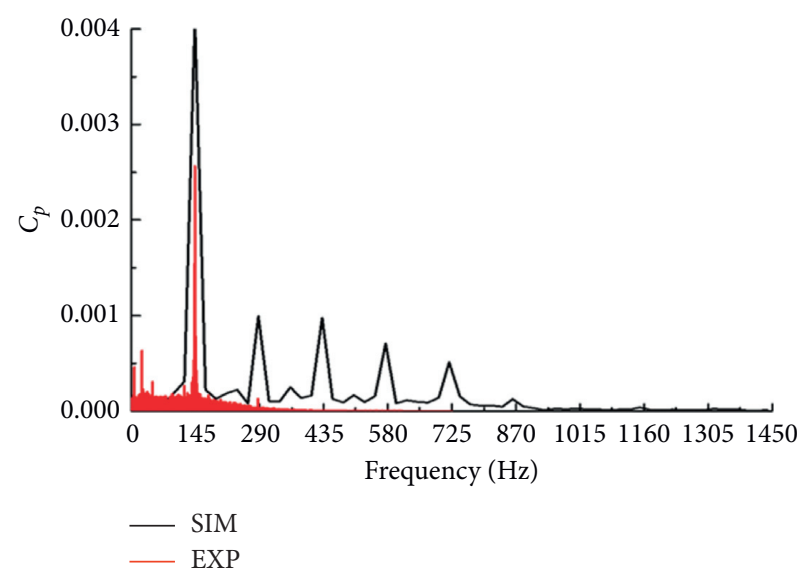

(b)

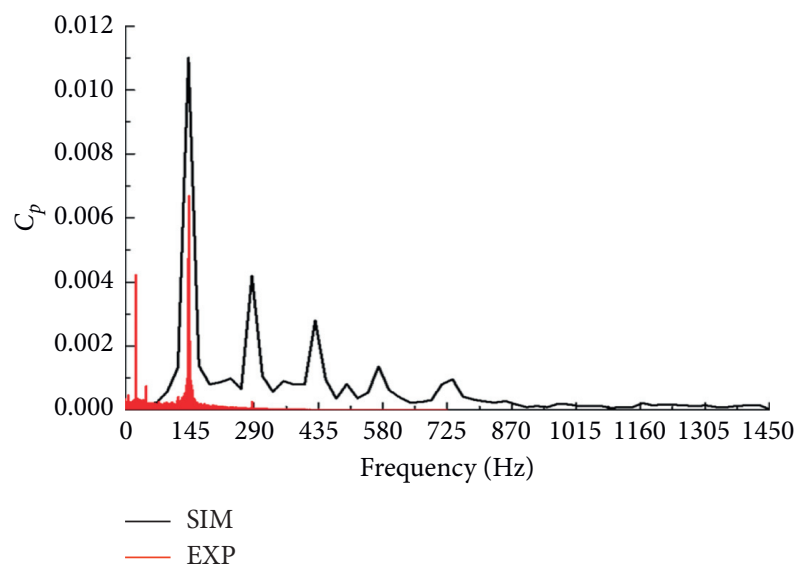

(d)

Figure 4: Comparison between the numerical simulation and the experiment of the pressure pulsation at $1.0 Q_{d}$. (a) $P 3$ time domain. (b) $P 3$ frequency domain. (c) P4 time domain. (d) P4 frequency domain.

Besides, the impeller is strongly affected by the rotor-stator interaction of the impeller-guide vane. The guide vanes are also affected by the impeller-volute and impeller-guide vane rotorstator interaction. The volute is strongly affected by the impeller-volute rotor-stator interaction. The interaction of the impeller-volute is mainly the interaction between the impeller and the volute tongue.

Figure 9 shows the entropy generation rate at three different moments in the volute at $48 \mathrm{~m}^{3} / \mathrm{h}$. The choices of three time periods are shown in the black vertical lines in Figure 8. High energy loss occurs near the volute tongue. After the fluid passes through the tongue, energy loss begins to occur on the wall of the spiral section of the volute and increases in the circumferential direction. Also, energy loss also occurs at the exit of the volute near the leading edge of the guide vane. Compared with the velocity and streamline distribution in the volute (see Figure 10), the flow field at the volute tongue is complicated. Since the trailing edge of the guide vane blade of the centrifugal pump was not rounded, the fluid will impact the guide vane vertically. Part of the fluid directly bounces back and impacts with the fluid in the spiral section of the volute. The two consecutive impacts complicate the flow field near the tongue and form two low-velocity zones and high-velocity gradients. This causes high energy loss at the volute tongue. In the spiral section of the volute, the wall of the volute forces the fluid to change the direction of velocity; in addition, the influence of the boundary layer resulted in a large flow velocity change and a high-velocity gradient near the volute wall, which caused high losses. The reduction of the cross-sectional area of the volute leads to a certain increase in the velocity gradient of the cross section of the volute, which caused increased losses. When the volute section is too small, the impact between the fluid and the guide vane blades will also become stronger, so obvious energy loss occurs at the guide vane blade DB2. There was no obvious change in the energy loss everywhere in the volute at different times, which shows that the rotor-stator interaction will not have a significant impact on the local loss in the volute.

Figure 11 depicts the distribution of entropy production on the guide vane at different times. The energy loss of the flow passage near the suction surface of the guide vane blade close to the tongue is significantly higher than that of other flow passages. Higher energy losses are also produced near the trailing edges of the 5 blades at the exit of the guide vane. 

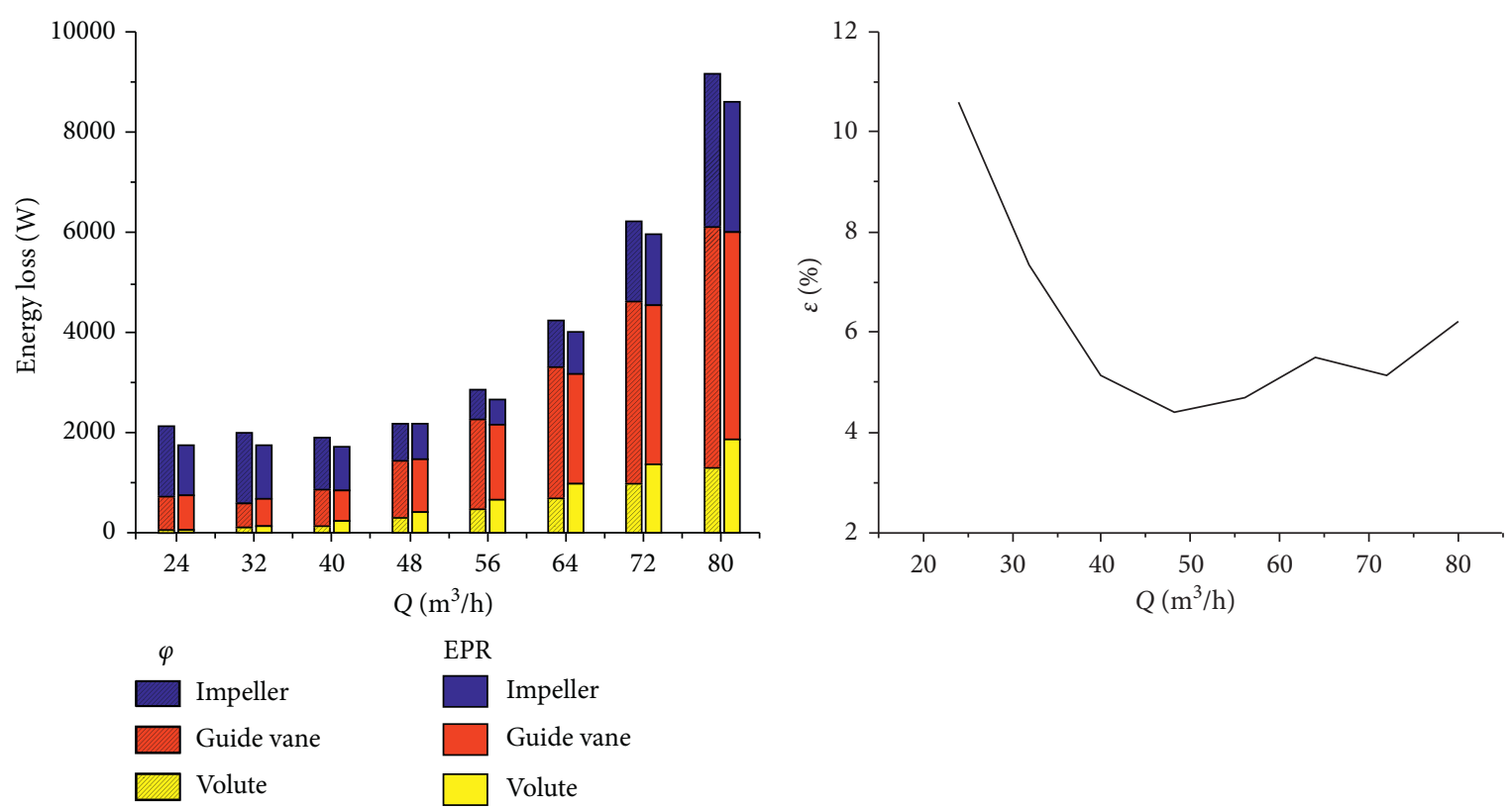

(a)

(b)

Figure 5: Comparison between loss calculated using the methods of entropy production and pressure difference. (a) Comparison of the two methods for calculating energy loss. (b) Error.

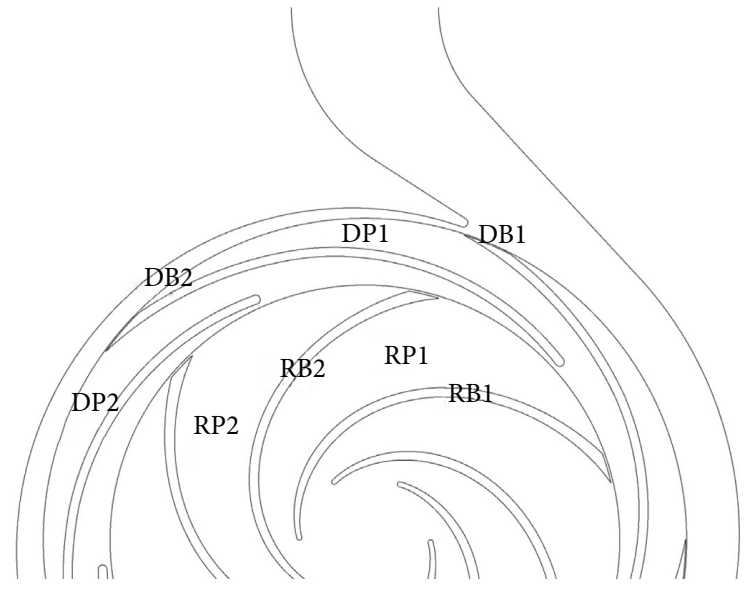

Figure 6: Naming of blades and passages.

Also, the energy loss near the wall of the guide vane blade DB2 is significantly higher than other blades. Due to the complex flow at the separation tongue, the liquid flow angle near the suction surface of the guide vane blade 1 is too large. The guide vane velocity distribution graph is shown in Figure 12. As a result, flow separation occurs at the leading edge of the suction force of the blade, and a wide range of low-speed areas appear, which affects the flow of the entire flow passage and causes a high energy loss on the suction surface of the blade DB1. The energy loss to the trailing edge of the blade is due to the round shape of the trailing edge of the blade, which easily forms a wake flow, which causes a high loss. The reduction of the flow cross-section of the upstream volute causes a certain difference between the angle of the fluid entering the flow channel and the angle of

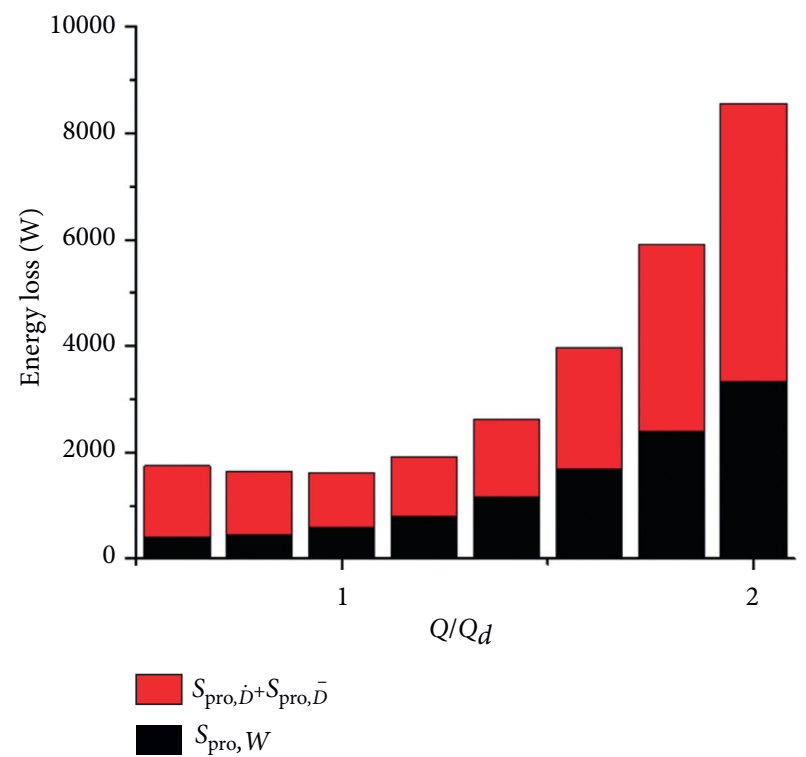

FIGURE 7: Total entropy production and wall loss under different flow conditions.

the blade, which results in a strong impact of the fluid on the blade and high energy loss.

The entropy production rate at the exit of the guide vane changes at different times, and the wake loss changes significantly. The energy loss near the volute has a little change within three moments. This is because when the impeller blades rotate to the vicinity of the wake, the transmission of the wake vortex will be blocked. When the wake hits the blade, the loss in the guide vane and impeller will increase. The blade inlet is far away from the impeller, so the rotation 

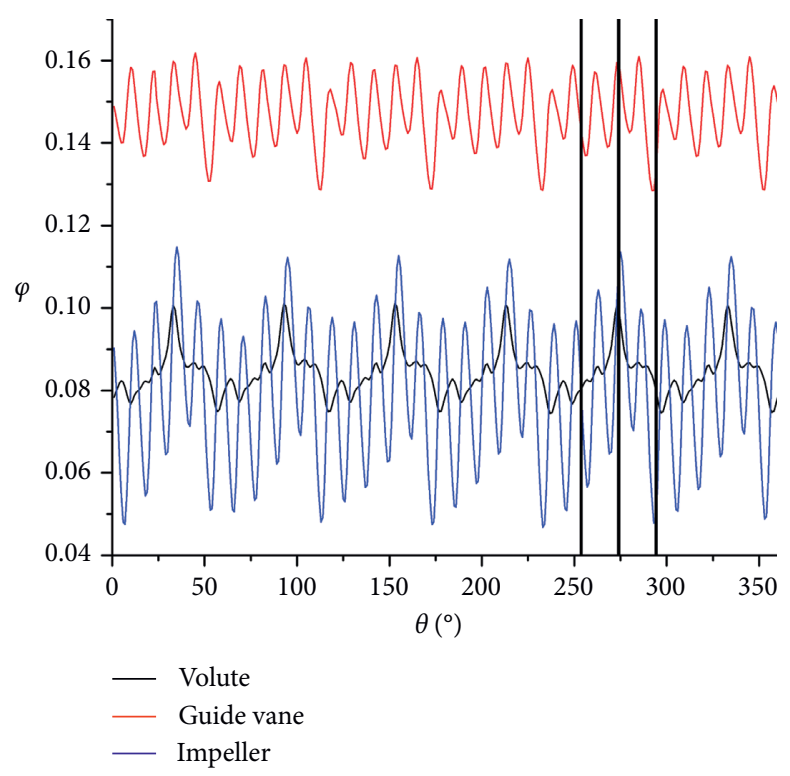

Figure 8: Energy loss of different parts inside PAT in one rotation cycle at $48 \mathrm{~m}^{3} / \mathrm{h}$.

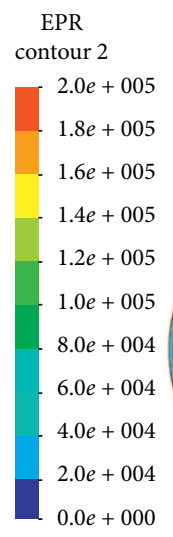

$\left(\mathrm{W} / \mathrm{m}^{-3}\right)$

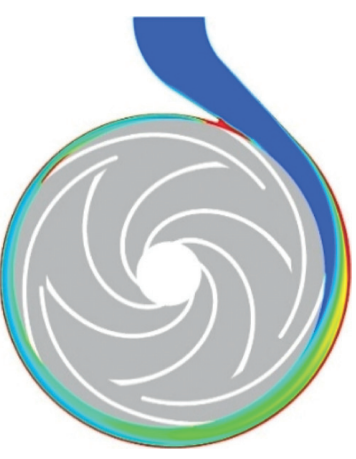

$T 1$

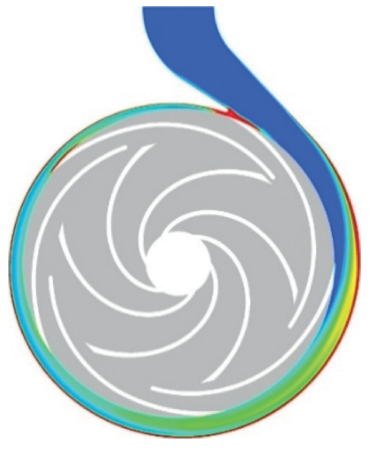

$T 2$

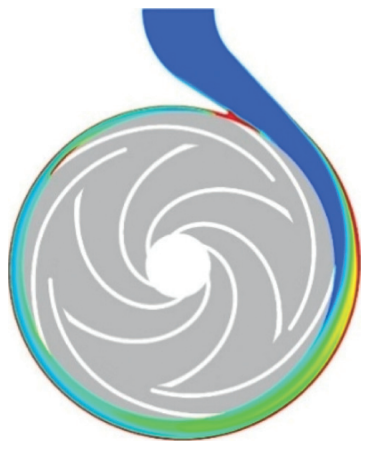

T3

FIGURE 9: Entropy production rate in different moments in volute at $48 \mathrm{~m}^{3} / \mathrm{h}$.
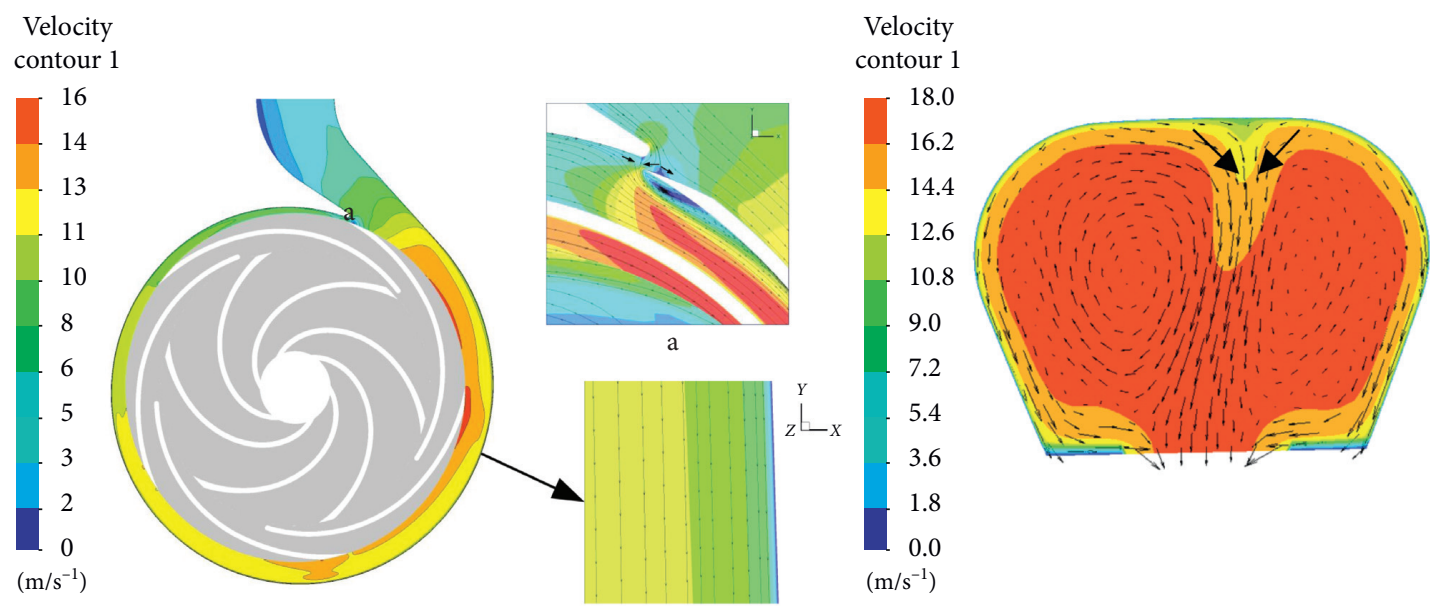

Figure 10: Streamlines in volute at $(Q)=48 \mathrm{~m}^{3} / \mathrm{h}$. 


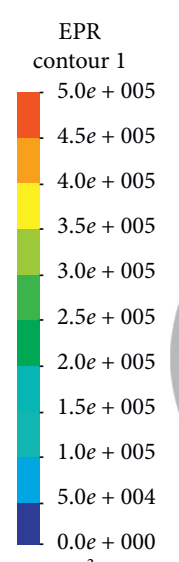

$\left(\mathrm{W} / \mathrm{m}^{-3}\right)$

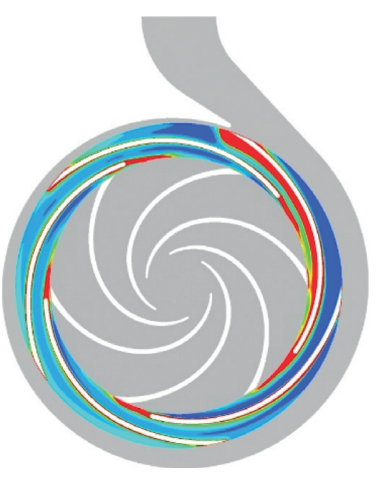

$T 1$

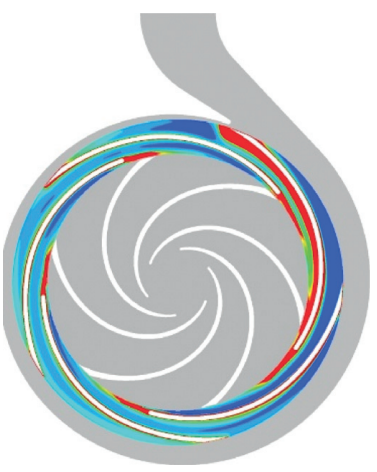

$T 2$

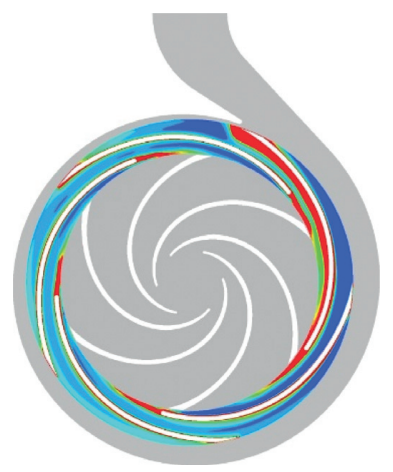

T3

Figure 11: Entropy production rate in different moments at $(Q)=48 \mathrm{~m}^{3} / \mathrm{h}$ in guide vane.

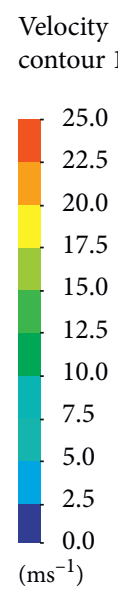

Velocity tour 1

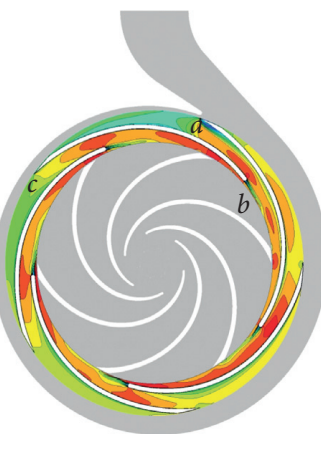

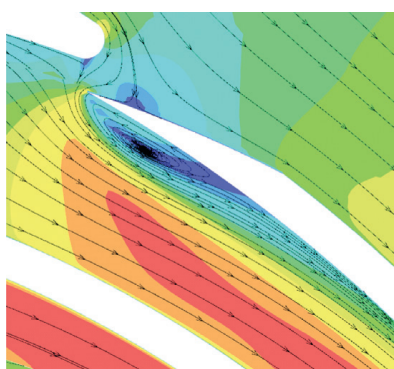

(a)

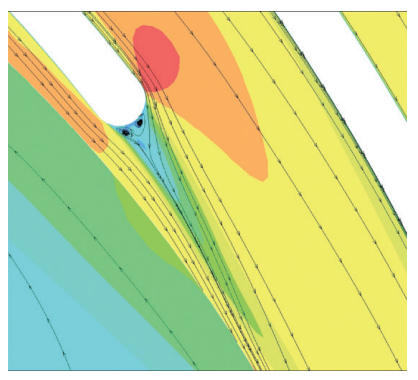

(b)

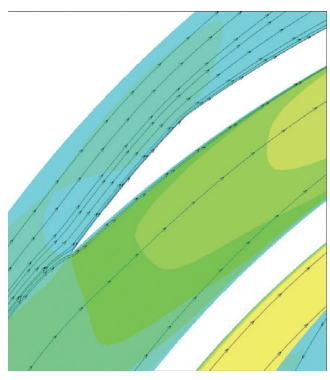

(c)

Figure 12: Streamlines in guide vane at $(Q)=48 \mathrm{~m}^{3} / \mathrm{h}$.

of the impeller has little effect on the energy loss of the guide vane inlet. This shows that the influence of the guide vane by the impeller-guide vane rotor-stator interaction is mainly caused by affecting the wake of the guide vane. Also, the loss at the guide vane channel DP1 has changed significantly at different times. This is because the fluid in the guide vane channel DP1 causes a strong impact on the impeller blades due to the influence of the volute tongue. This is the main reason why the guide vane is affected by the rotor-stator interaction of the impeller and the volute.

Figure 13 shows the energy loss distribution of different impeller spans at $T 2$ and $T 3$ under $Q=48 \mathrm{~m}^{3} / \mathrm{h}$. The losses in the impeller are concentrated near the entrance and exit of the impeller. And the energy loss near the shroud and the hub is significantly higher than the loss in the middle section. Thus, the impeller inlet is strongly affected by the rotor-stator interaction of the impeller-guide vane. In the streamline diagram (as shown in Figure 14), it was found that a large range of vortices appeared near the working surface of the impeller blades, causing nearly half of the impeller flow passage to be blocked, therefore, high energy loss near the impeller inlet. The loss at the exit of the impeller is caused by the blade wake flow. When it is close to the front and rear cover, the loss caused by the increased speed gradient is greater than the loss in the middle section. When the exit of the blade is close to the front cover, a wide range of low-speed zone appears, and the velocity distribution in the other two sections is more uniform. Therefore, the impeller outlet loss is the highest near the front cover. Although vortexes appear on the pressure surface, the loss of the pressure surface is lower than the energy loss of the suction surface due to the low flow velocity.

Comparing the loss in the impeller at different times, it can be seen that the position where the loss changes greatly is near the impeller inlet. The energy loss at the impeller inlet at $\mathrm{T} 2$ is significantly higher than the energy loss at the impeller inlet at T3. And the energy loss of the impeller flow path increases when passing through the guide vane flow path DP1. This shows that the flow in the impeller is affected by static and dynamic interference, is greatly affected by rotorstator interaction of the impeller-guide vane, and is less affected by the rotor-stator interaction. 


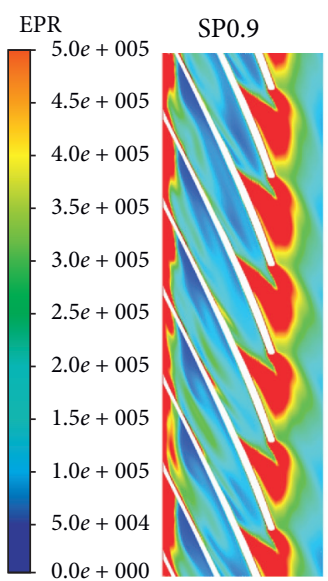

$\left(\mathrm{W} \mathrm{m}^{-3}\right)$
SP0.5

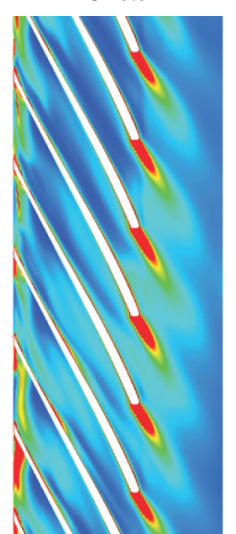

$T 2$
SP0.1

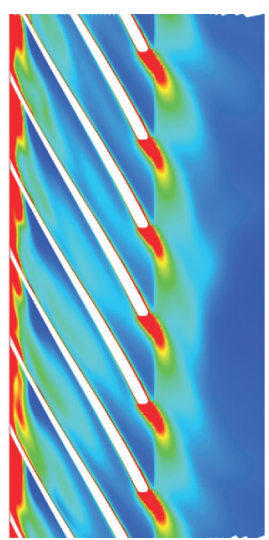

SP0.9

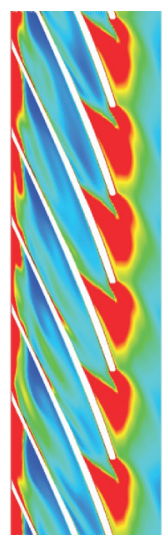

SP0.5

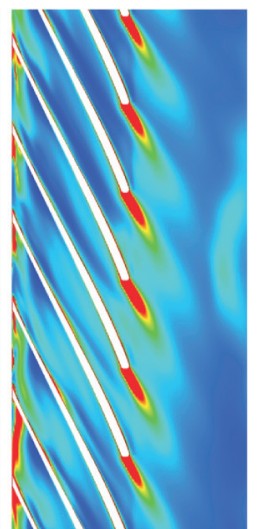

SP0.1

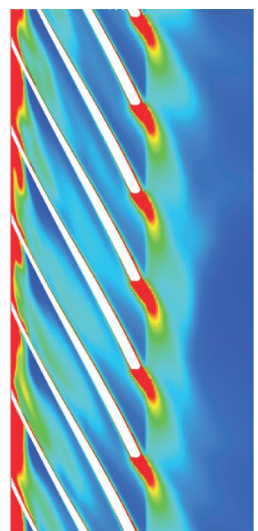

$T 3$

FIGURE 13: Entropy production distribution in different spanwise surfaces at $(Q)=48 \mathrm{~m}^{3} / \mathrm{h}$.

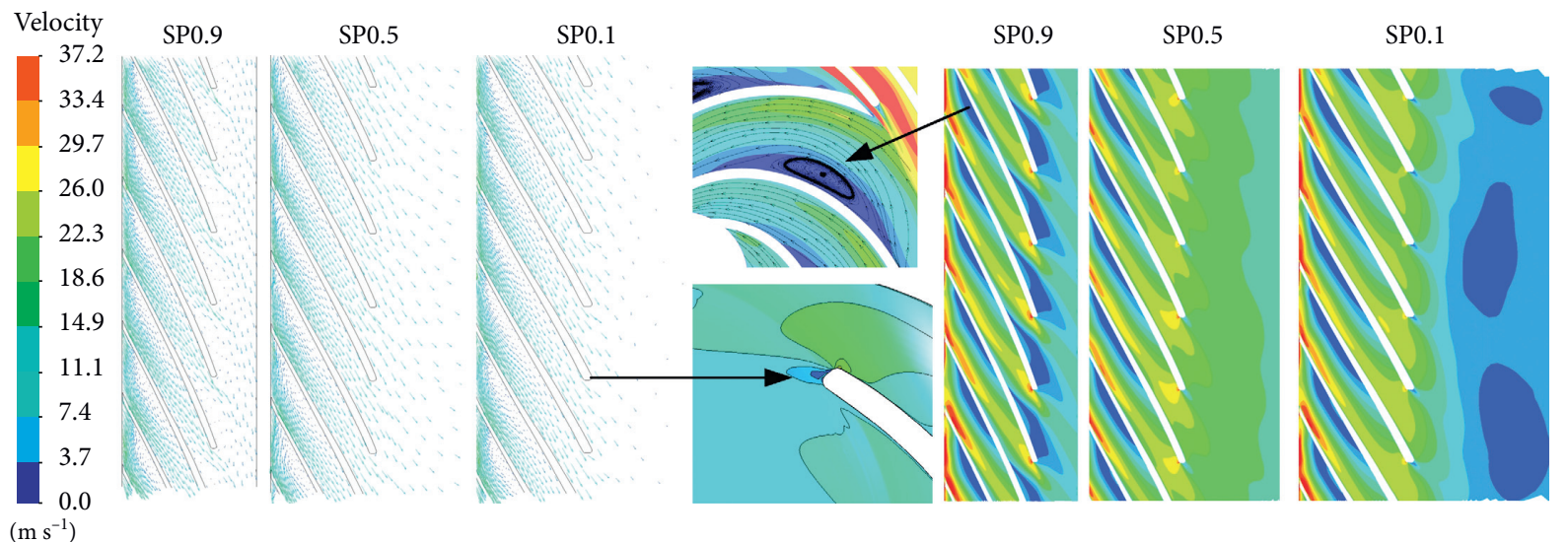

FIGURE 14: Streamlines in different spanwise surfaces at $(Q)=48 \mathrm{~m}^{3} / \mathrm{h}$.

\section{Conclusion}

This paper uses the entropy production theory to obtain the detailed situation of the loss caused by PAT during operation and analyzes the phenomenon of rotor-stator interaction in PAT. Find the following conclusions:

(1) The largest energy loss is the guide vanes, followed by the impellers, and the loss of the volute is relatively small in PAT. The asymmetric structure of the volute also affects the hydraulic loss of downstream components.

(2) The loss in the volute was mainly composed of friction loss and impact loss at the tongue. The loss in the guide vanes was due to the flow separation in the flow channel, the impact of the leading edge of the blade, and the blade wake. The loss in the impeller was mainly due to flow separation and wake flow.

(3) Rotor-stator interaction has the greatest influence on the vicinity of the interface between the impeller and the guide vane. The impeller is mainly affected by the rotor-stator interaction of the impeller and the guide vane, and the guide vane is affected by the rotorstator interaction of the impeller and the volute. Rotor-stator interaction is one of the main causes of PAT vibration. This study is beneficial to study the dynamic and static interference and PAT vibration to improve the stability of the PAT.

\section{Nomenclature}

BEP: Best efficiency point

CFD: Computation fluid dynamics

PAT: Pumps as turbines

$s: \quad$ Specific entropy

$\Phi: \quad$ Viscous dissipation term

$\Phi \Theta: \quad$ Dissipation term

T: $\quad$ Temperature (K)

$P_{\text {in,tp }}:$ Inlet pressure $(\mathrm{Pa})$

$P_{\text {out,tp }}$ : Outlet pressure $(\mathrm{Pa})$

$P_{\text {loss: }}$ Energy loss in the runner (W)

$\dot{S}_{\bar{D}}^{\prime \prime \prime}: \quad$ The entropy production by average velocity $\left(\mathrm{W} / \mathrm{m}^{3}\right)$

$\dot{S}_{D^{\prime}}^{\prime \prime \prime} \quad$ The entropy production by the pulsation velocity $\left(\mathrm{W} / \mathrm{m}^{3}\right)$ 
$S_{\text {pro }}: \quad$ Total entropy production (W)

$S_{\mathrm{pro}, \dot{D}}$ : The entropy production caused by the pulsation velocity (W)

$S_{\text {pro, }, \bar{D}}$ : The entropy produced by the time-averaged velocity (W)

$S_{\text {pro,w: }}$ The local entropy production rate for the wall

$\vec{v}: \quad$ Velocity vector $(\mathrm{m} / \mathrm{s})$

$\rho: \quad$ Density $\left(\mathrm{kg} / \mathrm{m}^{3}\right)$

$\varepsilon: \quad$ Turbulent dissipation rate $\left(\mathrm{m}^{2} / \mathrm{s}^{3}\right)$

$W_{s f t}: \quad$ Runner shaft power (W)

$\mu_{\text {eff: }} \quad$ Effective dynamic viscosity $\left(\mathrm{N} \cdot \mathrm{s} / \mathrm{m}^{2}\right)$

$\mu_{t}: \quad$ Turbulence dynamic viscosity $\left(\mathrm{N} \cdot \mathrm{s} / \mathrm{m}^{2}\right)$

$\stackrel{\vec{\tau}:}{\vec{\tau}:} \quad$ Wall shear stress $(\mathrm{Pa})$

$\varphi$ : $\quad$ Pressure drop coefficient

$Q_{d}: \quad$ Flow rate $\left(\mathrm{m}^{3} / \mathrm{s}\right)$

H: $\quad$ Head $(\mathrm{m})$

$n$ : $\quad$ Rotating speed (rev/min)

D1: $\quad$ Outer diameter of impeller $(\mathrm{mm})$

b1: $\quad$ Impeller blade width ( $\mathrm{mm})$

$z 1: \quad$ Number of impeller blades

D2: $\quad$ Diameter of guide vane inlet $(\mathrm{mm})$

D3: $\quad$ Diameter of guide vane outlet $(\mathrm{mm})$

b2: $\quad$ Guide vane blade width $(\mathrm{mm})$

$z 2$ : $\quad$ Number of guide vanes

D4: $\quad$ Diameter of volute base circle $(\mathrm{mm})$

$u_{2}$ : Circumferential velocity of impeller tail edge $(\mathrm{m} / \mathrm{s})$.

\section{Data Availability}

The data used to support the findings of this study are available from the corresponding author upon request.

\section{Conflicts of Interest}

The authors declare that they have no conflicts of interest.

\section{Acknowledgments}

This work was supported by the Scientific Research Program funded by the Shaanxi Provincial Education Department (Grant no. 20JK0957), the Natural Science Basic Research Plan in Shaanxi Province of China (Grant no. 2020JM-645), and Xijing University Special Research Foundation (Grant no. XJ17T09).

\section{References}

[1] K. Sengpanich, E. L. J. Bohez, P. Thongkruer, and K. Sakulphan, "New mode to operate centrifugal pump as impulse turbine," Renewable Energy, vol. 140, pp. 983-993, 2019.

[2] R. Tao, R. Xiao, F. Wang, and W. Liu, "Improving the cavitation inception performance of a reversible pump-turbine in pump mode by blade profile redesign: design concept, method and applications," Renewable Energy, vol. 133, pp. 325-342, 2019.

[3] M. Binama, W.-T. Su, W.-H. Cai et al., "Blade trailing edge position influencing pump as turbine (PAT) pressure field under part-load conditions," Renewable Energy, vol. 136, pp. 33-47, 2019.
[4] X. Li, H. Chen, B. Chen, X. Luo, B. Yang, and Z. Zhu, "Investigation of flow pattern and hydraulic performance of a centrifugal pump impeller through the PIV method," Renewable Energy, vol. 162, no. 12, pp. 561-574, 2020.

[5] Y. Hao and L. Tan, "Symmetrical and unsymmetrical tip clearances on cavitation performance and radial force of a mixed flow pump as turbine at pump mode," Renewable Energy, vol. 127, pp. 368-376, 2018.

[6] S. Abazariyan, R. Rafee, and S. Derakhshan, "Experimental study of viscosity effects on a pump as turbine performance," Renewable Energy, vol. 127, pp. 539-547, 2018.

[7] Y. Yang, L. Zhou, W. Shi, Z. He, Y. Han, and Y. Xiao, "Interstage difference of pressure pulsation in a three-stage electrical submersible pump," Journal of Petroleum Science and Engineering, vol. 196, p. 107653, 2021.

[8] A. Morabito and P. Hendrick, "Pump as turbine applied to micro energy storage and smart water grids: a case study," Applied Energy, vol. 241, pp. 567-579, 2019.

[9] L. Zhou, C. Han, L. Bai, W. Li, M. A. El-Emam, and W. Shi, "CFD-DEM bidirectional coupling simulation and experimental investigation of particle ejections and energy conversion in a spouted bed," Energy, vol. 211, p. 118672, 2020.

[10] X.-D. Lai, Q.-W. Liang, D.-X. Ye, X.-M. Chen, and M.-M. Xia, "Experimental investigation of flows inside draft tube of a high-head pump-turbine," Renewable Energy, vol. 133, pp. 731-742, 2019.

[11] A. Bozorgi, E. Javidpour, A. Riasi, and A. Nourbakhsh, "Numerical and experimental study of using axial pump as turbine in Pico hydropower plants," Renewable Energy, vol. 53, pp. 258-264, 2013.

[12] X. Su, S. Huang, X. Zhang, and S. Yang, "Numerical research on unsteady flow rate characteristics of pump as turbine," Renewable Energy, vol. 94, pp. 488-495, 2016.

[13] T. Wang, C. Wang, F. Kong, Q. Gou, and S. Yang, "Theoretical, experimental, and numerical study of special impeller used in turbine mode of centrifugal pump as turbine," Energy, vol. 130, pp. 473-485, 2017.

[14] Y. Liu and L. Tan, "Tip clearance on pressure fluctuation intensity and vortex characteristic of a mixed flow pump as turbine at pump mode," Renewable Energy, vol. 129, pp. 606-615, 2018.

[15] M. Liu, L. Tan, and S. Cao, "Theoretical model of energy performance prediction and BEP determination for centrifugal pump as turbine," Energy, vol. 172, pp. 712-732, 2019.

[16] S. Tang, S. Yuan, and Y. Zhu, "Deep learning-based intelligent fault diagnosis methods toward rotating machinery," IEEE Access, vol. 8, no. 1, pp. 9335-9346, 2020.

[17] S. Tang, S. Yuan, and Y. Zhu, "Convolutional neural network in intelligent fault diagnosis toward rotatory machinery," IEEE Access, vol. 8, no. 1, pp. 86510-86519, 2020.

[18] Y. Gao, X. Fan, and R. Dang, "Numerical characterization of the effects of flow rate on pressure and velocity distribution of pump as turbine," Current Science, vol. 117, no. 1, pp. 57-63, 2019.

[19] S.-c Miao, Z.-x Shi, X.-h Wang, F.-x Shi, and G.-t Shi, "Impeller meridional plane optimization of pump as turbine," Science Progress, vol. 103, no. 1, 2020.

[20] H. Hou, Y. Zhang, Z. Li, T. Jiang, J. Zhang, and C. Xu, "Numerical analysis of entropy production on a LNG cryogenic submerged pump," Journal of Natural Gas Science and Engineering, vol. 36, pp. 87-96, 2016.

[21] C. Wang, Y. Zhang, H. Hou, J. Zhang, and C. Xu, "Entropy production diagnostic analysis of energy consumption for cavitation flow in a two-stage LNG cryogenic submerged pump," 
International Journal of Heat and Mass Transfer, vol. 129, pp. 342-356, 2019.

[22] G. Hou, H. Taherian, L. Li, J. Fuse, and L. Moradi, "System performance analysis of a hybrid ground source heat pump with optimal control strategies based on numerical simulations," Geothermics, vol. 86, no. May, p. 101849, 2020.

[23] G. Hou, H. Taherian, and L. Li, "A predictive TRNSYS model for long-term operation of a hybrid ground source heat pump system with innovative horizontal buried pipe type," Renewable Energy, vol. 151, pp. 1046-1054, 2020.

[24] S. V. Jain and R. N. Patel, "Investigations on pump running in turbine mode: a review of the state-of-the-art," Renewable and Sustainable Energy Reviews, vol. 30, pp. 841-868, 2014.

[25] H. Herwig and F. Kock, "Direct and indirect methods of calculating entropy generation rates in turbulent convective heat transfer problems," Heat and Mass Transfer, vol. 43, no. 3, pp. 207-215, 2006.

[26] D. Li, H. Wang, Y. Qin, L. Han, X. Wei, and D. Qin, "Entropy production analysis of hysteresis characteristic of a pump-turbine model," Energy Conversion and Management, vol. 149, pp. 175-191, 2017.

[27] G. Peng, X. Huang, L. Zhou, G. Zhou, and H. Zhou, "Solid-liquid two-phase flow and wear analysis in a large-scale centrifugal slurry pump," Engineering Failure Analysis, vol. 114, p. 104602, 2020. 